\title{
Transformations of Axes for Whittaker's Solution of Laplace's Equation.
}

\author{
By G. B. J JFFkry, University College, London.
}

(Received 15th February 1917. Read 9th March 1917.)

Whittaker has shown that a general solution of Laplace's equation, $\nabla^{2} V=0$, may be expressed in the form

$$
V=\int_{0}^{3 \pi} f(i x \cos t+i y \sin t+z, t) d t .
$$

Since the harmonic property of a function is in no way dependent upon any particular set of axes it follows that the same solution must be capable of being expressed in the form

$$
V=\int_{0}^{2 \pi} F(i X \cos T+i Y \sin T+Z, T) d t
$$

where $X, Y, Z$ are any second set of rectangular coordinates.

The object of the present paper is to exhibit the solution between the functions $f, F$ in a fairly wide class of case. The analysis is, for the most part, quite straightforward, and we will only give it in outline.

Consider the most general transformation of orthogonal axes having the same origin. Then

$$
\begin{aligned}
& x=l_{1} X+m_{1} Y+n_{1} Z \\
& y=l_{2} X+m_{2} Y+n_{2} Z \\
& z=l_{3} X+m_{3} Y+n_{3} Z
\end{aligned}
$$

where the direction cosines satisfy the usual orthogonal relations, and, if both sets of axes are right-handed, there is also a number of relations of which $l_{1}=m_{2} n_{3}-m_{2} n_{2}$ is the type. 
Then

Let

$$
\begin{aligned}
i x \cos t+i y \sin t+z & =i X\left(l_{1} \cos t+l_{2} \sin t-i l_{3}\right) \\
& +i Y\left(m_{1} \cos t+m_{2} \sin t-i m_{3}\right) \\
& +Z\left(i n_{1} \cos t+i n_{2} \sin t+n_{3}\right) .
\end{aligned}
$$

$$
\frac{l_{1} \cos t+l_{2} \sin t-i l_{3}}{i n_{1} \cos t+i n_{2} \sin t+n_{3}}=\cos T
$$

and

$$
\frac{m_{1} \cos t+m_{2} \sin t-i m_{3}}{i n_{1} \cos t+i n_{2} \sin t+n_{3}}=\sin T .
$$

These are consistent and completely define $T$, if we suppose that when $t=0,0 \leq$ real part of $T<2 \pi$.

We readily obtain the following relations :-

$$
\begin{gathered}
\frac{l_{1} \cos T+m_{1} \sin T-i n_{1}}{i l_{3} \cos T+i m_{8} \sin T+n_{3}}=\cos t \quad \ldots \ldots \ldots \ldots \ldots \\
\frac{l_{2} \cos T+m_{2} \sin T-i n_{2}}{i l_{3} \cos T+i m_{3} \sin T+n_{3}}=\sin t \quad \ldots \ldots \ldots \ldots \ldots \\
\left(n_{1} \cos t+n_{2} \sin t-i n_{3}\right)\left(l_{3} \cos T+m_{3} \sin T-i n_{3}\right)=-1
\end{gathered}
$$

and

$$
\frac{d t}{d T}=i n_{1} \cos t+i n_{2} \sin t+n_{3}
$$

Hence

$$
\begin{aligned}
& \int_{0}^{2 \pi} f(i x \cos t+i y \sin t+z, t) d t \\
& =\int_{C} f\left(\frac{i X \cos T+i Y \sin T+Z}{i l_{3} \cos T+i m_{3} \sin T+n_{3}}, t\right) \frac{d T}{i l_{3} \cos T+i m_{3} \sin T+n_{3}} \ldots
\end{aligned}
$$

where, in the second integral, $t$ is to be expressed in terms of $T$ by (3) and (4), and $C$ is a certain contour in the $T$-plane.

In order to investigate the nature of this contour, let us put $T=U+i V$ and solve (1) and (2) for $U, V$. We obtain

and

$$
\begin{aligned}
& \sin U=-\frac{l_{2} \cos t-l_{1} \sin t}{\sqrt{ }\left\{1-\left(n_{2} \cos t-n_{1} \sin t\right)^{2}\right\}} \\
& \tan U=-\frac{l_{2} \cos t-l_{\mathrm{t}} \sin t}{m_{2} \cos t-m_{1} \sin t} \quad \ldots \ldots \ldots \ldots \ldots
\end{aligned}
$$

$$
\tanh V=n_{2} \cos t-n_{1} \sin t \text {. }
$$


The curve corresponding to real values of $t$ is shown in the diagram.

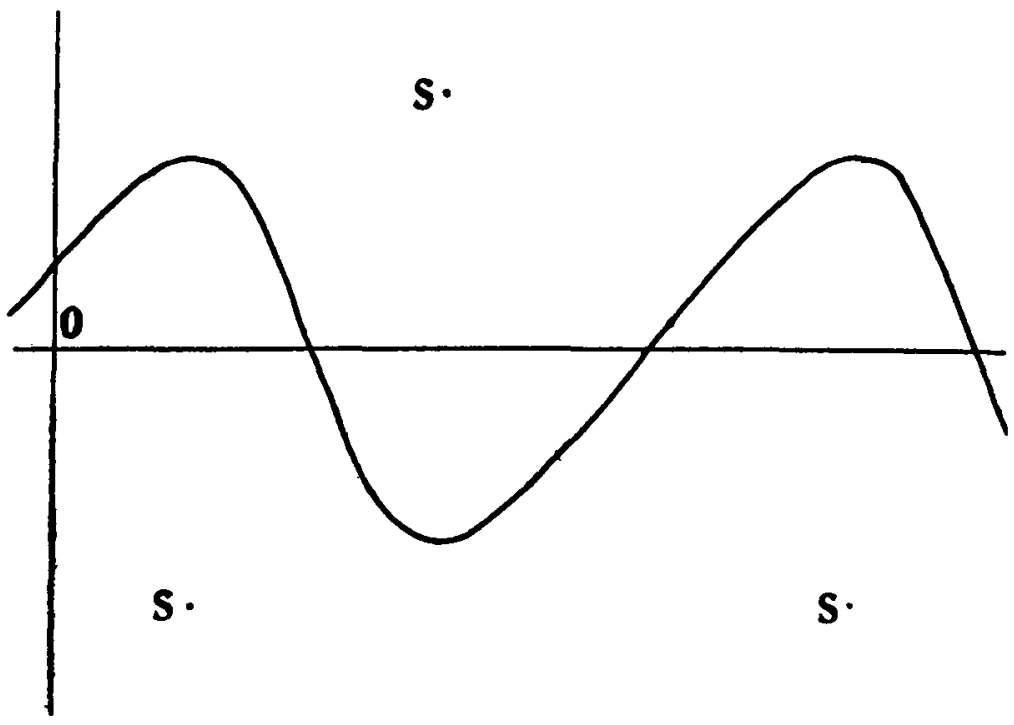

The $T$-plane showing the curve corresponding to resl values of $t$ and the zeros of $\dot{u}_{3} \cos T+i m_{3} \cos T+n_{3}$.

The maximum ordinates are given by $\tanh V=\sqrt{ }\left(1-n_{3}{ }^{2}\right)$ and correspond to $\tan t=-n_{1} / n_{2}$. The curve cuts the axis at points corresponding to $\tan t=n_{1} / n_{2}$. The contour $C$ will then be a complete period of this curve.

If the function $f$ is periodic in $t$ with period $2 \pi$, it will be periodic in $T$ with the same period. We may then take any complete period of the curve for the contour $C$.

If, in addition, the integrand, regarded as a function of $T$, has no singularities in the area contained between the curve and the real axis of $T$, the integration may be taken along the real axis from 0 to $2 \pi$. If $f$ is a one-valued function of $T^{\prime}$ in the area between the curve and the real axis, poles in this region will not invalidate the argument if the sum of the residues at the poles in that part of this region which lies above the axis is equal to the sum of the residues at the poles in the part below the axis. 
With these restrictions we may write

$$
\begin{aligned}
& \int_{0}^{2 \pi} f(i x \cos t+i y \sin t+z, t) \\
& =\int_{0}^{2 \pi} F\left(\frac{i X \cos T+i Y \sin T+Z}{i l_{3} \cos T+i m_{3} \sin T+n_{3}}, t\right) \frac{d T}{i l_{3} \cos T+i m_{3} \sin T+n_{3}} .
\end{aligned}
$$

Without making an exhaustive investigation of the singularities of the integrand, we may examine some of the more important possibilities.

First consider the zeros of $i l_{3} \cos T+i m_{3} \sin T+n_{3}$. Let such a zero occur at the point $T=u+i v$. Then we have without difficulty

$$
\tan u=-\frac{l_{3}}{m_{3}}, \sinh v= \pm \frac{n_{3}}{\sqrt{ }\left(1-n_{3}^{2}\right)} .
$$

From these it may be shown that these zeros lie on the ordinates through the maxima and the minima of the curve and on the opposite side of the real axis from the curve. These points are marked $\mathbf{S}$ in the diagram. Singularities of the integrand arising from these zeros therefore cannot invalidate the deformation of the contour.

Singularities of the integrand may occur at the zeros of $i X \cos T+i Y \sin T+Z$. At such a point let $T=u+i v$, then we have

$$
\begin{gathered}
\sin u= \pm \frac{X}{\sqrt{\left(x^{2}+y^{2}\right)}}, \cos u=\mp \frac{Y}{\sqrt{\left(x^{2}+y^{2}\right)}} \\
\sin v=\mp \frac{Z}{\sqrt{\left(x^{2}+y^{2}\right)}} .
\end{gathered}
$$

Let $T=u+i V$ be the point on the curve having the same abscissa, then from (8)

$$
\tan u=-\frac{X}{Y}=-\frac{l_{2}-l_{1} \tan t}{m_{2}-m_{1} \tan t}
$$

or

$$
\tan t=\frac{m_{2} X-l_{2} Y}{m_{1} X-l_{1} Y}
$$

From (7), (8), and (9) we then obtain

$$
\frac{\sinh V}{\sinh v}=-\frac{l_{3} X+m_{3} Y}{n_{3} Z}
$$


Hence the condition that the zeros of $i X \cos T+i Y \sin T+Z$ shall not lie between the curve and the real axis is

that is,

$$
\frac{l_{3} X+m_{3} Y}{n_{3} Z}>-1
$$

$$
\frac{z}{n_{3} Z}>0
$$

or the positive directions of the axes of $z, Z$ make an acute angle with each other.

In some cases it is sufficient to consider a less general transformation of axes. Let $\chi$ be the angle between the axes of $z$ and $Z$, and let the axis of $X$ be in the plane $z Z$, then the axis of $Y$ coincides with the axis of $y$. Then

$$
\begin{aligned}
l_{1,2,3}=\cos \chi, & 0, \quad-\sin \chi \\
m_{1,2,3}=0, & 1, \quad 0 \\
n_{1,2,3}=\sin \chi, & 0, \quad \cos \chi,
\end{aligned}
$$

The curve in the T-plane which corresponds to real values of $t$ then passes through the origin, and the contour $C$ begins and ends on the real axis. It is, therefore, unnecessary to make any assumption as to the periodicity of $f$. Our result then becomes

$$
\begin{aligned}
\int_{0}^{2 \pi} f(i x \cos t+i y \sin t+z, t) d t \\
\quad=\int_{0}^{2 \pi} f\left(\frac{i X \cos T+i Y \sin T+Z}{\cos \chi-i \sin \chi \cos T}, t\right) \frac{d T}{\cos \chi-i \sin \chi \cos T},
\end{aligned}
$$

where the assumptions as to the singularites of $f$ are the same as before. The condition that no zero of $i X \cos T+i Y \sin T+Z$ shall lie between the curve and the real axis is now $\chi<\frac{1}{2} \pi$.

As an example, let us consider the zonal spherical harmonics given by

$r^{n} P_{n}(\cos \theta)=\frac{1}{2 \pi} \int_{0}^{2 \pi}(i x \cos t+i y \sin t+z)^{n} d t$

$$
=\frac{1}{2 \pi} \int_{0}^{2 \pi} \frac{(i X \cos T+i Y \sin T+Z)^{n}}{(\cos \chi-i \sin } \frac{x \cos T)^{n+1}}{(T)}
$$


Now if $\chi<\frac{1}{2} \pi$, a simple application of the integral of Laplace's type for $P_{n}^{m}$ gives

$(\cos \chi-i \sin \chi \cos T)^{-n-1}=P_{n}(\cos \chi)+2 \sum_{m=1}^{\infty} \frac{\Gamma(n-m+1)}{i^{m} \Gamma(n+1)} P_{n}^{m}(\cos \chi) \cos m T$.

Hence

$$
\begin{aligned}
& r^{n} P_{n}(\cos \theta)=\frac{1}{2 \pi} P_{n}(\cos \chi) \int_{0}^{2 \pi}(i X \cos T+i Y \sin T+Z)^{n} d T \\
& +\frac{1}{\pi} \sum_{1}^{\infty} \frac{\Gamma(n-m+1)}{i^{m} \Gamma(n+1)} P_{n}^{m}(\cos \chi) \int_{0}^{2 \pi}\left(i X \cos T^{\prime}+i Y \sin T+Z\right)^{n} \cos m T^{\prime} d T
\end{aligned}
$$

Now*

$$
\begin{aligned}
\int_{0}^{2 \pi}(i X \cos T+ & i Y \sin T+Z)^{n} \cos m T d T \\
& =\frac{2 \pi i^{m} \Gamma(n+1)}{\Gamma(n+m+1)} r^{n} P_{n}^{m}(\cos \theta) \cos m \Phi
\end{aligned}
$$

where $\theta, \Phi$ are spherical polar coordinates referred to the axis of $\boldsymbol{Z}$ and the plane $X \boldsymbol{Z}$.

Hence we have at once the well-known "addition formula"

$$
\begin{aligned}
P_{n}(\cos \theta)= & P_{n}(\cos \chi) P_{n}(\cos \theta) \\
& +2 \sum_{m=1}^{\infty} \frac{\Gamma(n-m+1)}{\Gamma(n+m+1)} P_{n}^{n n}(\cos \chi) P_{n}^{m}(\cos \theta) \cos m \Phi .
\end{aligned}
$$

It appears that in a fairly wide class of cases the transformation of Whittaker's integral presents no difficulty. This investigation, however, leaves untouched the wider problem which arises when the conditions, which we have postulated above, as to periodicity and the absence of singularities between the real axis and the curve of integration are not fulfilled. In these cases also the integral, though of course not necessarily the integrand, must transform.

The author is indebted to Professor Whittaker for calling his attention to the problem, towards the solution of which this paper is intended as a contribution.

* Whittaker and Watson, Modern Analysis, p. 385. 